\title{
Research on Specification for Dedicated Short Range Communication
}

\author{
Tien-Chen, Ho \\ Dept. of Information Technology \\ Overseas Chinese University \\ Taichung, Taiwan \\ tcho@ocu.edu.tw
}

\begin{abstract}
Dedicated Short-Range Communication (DSRC) technology has various applications, such as Intelligent transport systems (ITS), Electronic toll collection (ETC) and Camel-Vehicle-Accident-Avoiding-System (CVAAS), etc.. The ETC has been used in many countries, such as USA, Malaysia, Thailand and Japan [9]. In the United States, ETC has been shown to increase $250 \%$ dealing than human toll collection. DSRC provides the platform for interoperable ETC and access control of various applications. This research points out the DSRC specification can readily meet the market need for stability and interoperability.
\end{abstract}

Keywords-Dedicated Short-Range Communication (DSRC), Electronic toll collection (ETC), On Board Equipment (OBE), Road side equipment (RSE)

\section{INTRODUCTION}

DSRC technology has various applications, such as ITS, ETC and CVAAS, etc..CVAAS can identify the presence of a camel on or near the highway for a distance of $100-300 \mathrm{~m}$ around the Road Side unit (RSU) and then the programmable GPS device will send out a signal to the DSRC transmitter. It stores the highway map and the description of the dangerous zones [6]. The key technology of vehicle-to-vehicle (V2V) is using DSRC or IEEE 802.11p also called WAVE (Wireless Access in the Vehicular Environment). The Spectrum allocation of Vehicle-Infrastructure Integration (VII) WAVE / DSRC is shown in figure 1 [8]. The interoperability between various applications is essential and requires the specification of precise technical characteristics.

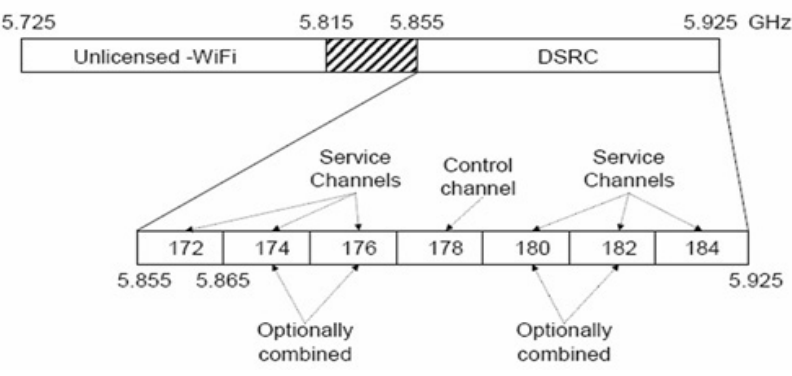

Figure 1. Spectrum allocation of VII WAVE/DSRC

\section{TECHNICAL CHARACTERISTICS FOR DSRC}

A. The Active Angle On Board Equipment (OBE) are :

-horizontally: $\pm 25^{\circ}$ with respect to the vertical plane through the longitudinal axis of the vehicle. -vertically: $35^{\circ}<\mathrm{A}<80^{\circ}$, where $\mathrm{A}$ is the angle with respect to the horizontal plane.

\section{B. Downlink (DL) / uplink (UL) Parameters}

In Europe, there are four allowed carrier frequencies (D1) for the DL channel: $5.7975 \mathrm{GHz}, 5.8025 \mathrm{GHz}, \quad 5.8075$ $\mathrm{GHz}, 5.8125 \mathrm{GHz}$.

The deviation of the carrier frequency from its nominal value is less than $\pm 5 \mathrm{ppm}$ for all operating conditions (D1a). The OBEMinimum Receiver Bandwidth is $5.795-5.815$ $\mathrm{GHz}$ (D3). The RSE Transmitter Spectrum Mask (D2). The emitted equivalent isotropically radiated power (EIRP) is less than $-30 \mathrm{dBm}$ outside the used frequency band $5.795-$ $5.815 \mathrm{GHz}[4]$. For in-band emissions the RSE transmitter spurious emissions must comply with at least one of the classes. Co-channel ULs are the UL frequencies at $\mathrm{fc} \pm 1.5$ $\mathrm{MHz}$ and $\mathrm{fc} \pm 2.0 \mathrm{MHz}$, where $\mathrm{fc}$ is the utilized carrier frequency. Adjacent channel ULs are the UL frequencies at $\mathrm{fa} \pm 1.5 \mathrm{MHz}$ and $\mathrm{fa} \pm 2.0 \mathrm{MHz}$, where fa is the remaining carrier frequencies. The maximum EIRP of the RSE transmitter is less than $+33 \mathrm{dBm}$ (D4). The RSE transmitter must keep the EIRP below $+18 \mathrm{dBm}$ for angles larger than $70^{\circ}$ with respect to the vertical axis (D4a). The main part of the emitted power is directed towards the ground. The polarization is left hand circular (D5/ U5), i.e. when looking in the direction of wave propagation, the tip of the electrical vector shall rotate anti-clockwise. The requirements below are applicable to the equipment itself not including other external effects. The Cross Polarization Discrimination (D5a/ U5a), i.e. the residual right hand circular polarized wave is lower than the expected polarization by a factor not less than: for RSE transmission/ reception in the boresight direction: $>15 \mathrm{~dB}$

for $\mathrm{OBE}$ reception/ transmission in the bore sight direction: $>10 \mathrm{~dB}$

for RSE transmission/ reception within the OBE Active Angle: $>10 \mathrm{~dB}$

for $\mathrm{OBE}$ reception/ transmission within the $\mathrm{OBE}$ Active Angle: $>6 \mathrm{~dB}$

A two-level amplitude modulation is used, Modulation and Eye Pattern (D6) ensure to comply with the RSE. In Transmitter Spectrum Mask, the modulation index (D6a) is defined as $(V \max -\mathrm{Vmin}) /(\mathrm{Vmax}+\mathrm{Vmin})$, and within the range 0.5 - 0.9. $\mathrm{Vmax} / \mathrm{Vmin}=\operatorname{maximum} / \mathrm{minimum}$ amplitude during modulation. The eye pattern (D6b) defines the free decision distance of a digital signal pulse with respect to pulse width and amplitude. An ideal digital signal has a decision height of $100 \%$ which is equal to the difference of high level and low level. Considering bi-phase 
coding, the ideal (=100\%) distance in width is equal to half the bit duration. The eye pattern requirements are:

Pulse width : 2B' / $\left(\mathrm{A}^{\prime}+\mathrm{B}^{\prime}\right)>0.90$, Pulse amplitude : $2 \mathrm{~B} /(\mathrm{A}+\mathrm{B})>0.85$

The bits are coded(D7) in FM0, i.e. there is always a transition at the beginning and end of the bit interval.

-A " 0 " bit has an additional transition in the middle of the bit interval.

-A "1" has no such additional transition in the middle of the bit interval.

The DL bit rate (D8) is $500 \mathrm{kBit} / \mathrm{s} \pm 100 \mathrm{ppm}$. The communication zone is defined as the volume in space where the reference bit error rate (BER) for Communication (D11, D9a) equal to or lower than 10-6 can be achieved.

The UL data coding (U7) is Non return to zero inverted (NRZI), i.e.:

-there is always a transition at the beginning of a " 0 " bit.

-there is no transition at the beginning of a " 1 " bit. -the level is kept constant for the whole bit duration.

The UL bit rate (U8) is $250 \mathrm{kbit} / \mathrm{s} \pm 0.1 \%$. The modulation of the subcarrier (U6a) is binary phase shift keying (BPSK) which.uses bi-phase $\left(0^{\circ}, 180^{\circ}\right)$. A phase shift of the subcarrier is generated at each transition of the UL encoded data

\section{Protocol Data Units (PDU)}

The Link PDU (LPDU) consists of Logical Link Control (LLC) control field, LLC status field and Application PDU (APDU). The frame is read from left to right, i.e. the leftmost field is transmitted first. The frame format is similar for the DL and for the UL. The DL carries only Data Link Layer (DLL) command frames, while the UL can carry DLL command and response frames. Each frame does not necessarily contain all of the fields depicted above. The most notable exceptions are

-Only response frames contain the LLC status field,

-Response frames do not always contain an LPDU information field,

-There are frames without LPDU (used for medium request / allocation).

The size of the frame, including the flags and anything between the flags but excluding the preamble is subject to the following restrictions:

-in a DL window max 128 octets (N2),

-in a private UL window max 128 octets (N3)

-in a public UL window max 9 octets (N4).

The number of bits transmitted is dependant on bit stuffing. The flag is an eight bit sequence, namely 01111110 , delimiting the content of the frame. Any sequence of 0111 1110 is interpreted as a valid flag sequence.

\section{Link ID (LID)}

All DSRC layers and the application inside RSE make use of this private Link ID (LID). The applications inside RSE use the private LID to access data inside a specific OBE. The Broadcast LID is one octet with all bits ' 1 '. The Private LID is four octets with all bits but the least significant bit (LSB) 's are randomly chosen by the OBE:

$$
\text { x X x x x x x } 0 \text { octet \#1 }
$$

$\mathrm{x} \times \mathrm{x} \times \mathrm{x} \times \mathrm{x} 0$ octet $\# 2$

x $\times$ x $\times$ x $\times$ x 0 octet \#3

x $\times$ x $\times$ x $\times$ x 1 octet \#4

MSB LSB, ' $x$ ' shall be $0 / 1$.

The one octet MAC control field is used to:

-indicate whether an LLC unit is available (to allow also for empty LPDU frames),

-indicate the transmission direction: DL or UL,

-allocate public and private UL windows,

-request private UL window, -specify type of LLC unit.

\section{E. Time constraints}

The start of the first private / public UL window occurs 160 us (T3) after the end of the DL window containing the frame allocating the private/ public UL window. The end of a private UL window occurs 320 us (T4a) after the start of the window, if no OBE has started transmitting the first bit of its preamble before that time or otherwise at the end of the last bit of the end flag of the UL frame transmitted. The maximum time of a private UL window is $5.5 \mathrm{~ms}$. Three consecutive windows are simultaneously allocated. The start of subsequent public UL windows occurs immediately after the end of the previous window. The end of a public window occurs 448 us (T5) after the start of that window.

\section{F. DSRC Profile}

Each set of parameter values is called a DSRC Profile and is assigned a unique identifier number. The profiles 0 and 1 as defined in [7] are supported. The choice of profile is primarily the responsibility of the RSE. All OBE shall support both profiles. The important parameter values of the profiles 0 and 1 are listed below.

Parameter

Profile

number $0 \quad 1$

D8 DL bit rate

$500 \quad 500 \mathrm{kBit} / \mathrm{s}$

U1 sub-carrier frequency

U8 UL symbol (bit) rate $1.5 \quad 2.0 \mathrm{MHz}$

$250250 \mathrm{kBit} / \mathrm{s}$

N2 max no of octets in frame in DL window 128128

N3 max no of octets in frame in private UL window 128128

N4 max no of octets in frame in public UL window 99

N5 no of simultaneously allocated public UL windows 33

N12 maximum private medium response time $1 \quad 1$

T1 minimum time after UL window that a DL window can start $32 \quad 32$ us

T2 minimum DL to DL window time $0 \quad 0$ us

$\mathrm{T} 3 \mathrm{DL}$ to UL turn around time 160160 us

T4a max time to start of transm. in private UL window 320320 us 
T4b max time to start of transm. in public UL window 3232 us

T5 time duration of public UL window 448448 us

N13FE acknowledgement time of fixed equipment 11

N13ME acknowledgement time of mobile equipment 11

Before any two-way information exchange (DL and UL) is performed, we must start the uniformed initialization procedure which can establish interoperable communication to transmit the private LID to the RSE and transmit the Vehicle Service Table (VST) to the RSE. The standards for DSRC contain a number of options and alternatives on each layer [1], [2] ,[3].

\section{G. State Transition Table}

There are total 68 reference numbers (Ref no.) and 68 Comments on the transitions in the State Transition Table. We just list the first two Ref no. for reference.

TABLE I. State Transition TABle

\begin{tabular}{|c|c|c|c|c|}
\hline $\begin{array}{c}\text { Curr } \\
\text { ent } \\
\text { State }\end{array}$ & $\begin{array}{c}\text { Ref } \\
\text { no. }\end{array}$ & Event & Action(s) & $\begin{array}{c}\text { Next } \\
\text { State }\end{array}$ \\
\hline $\begin{array}{c}\text { WAI } \\
\text { T }\end{array}$ & 1 & $\begin{array}{c}\text { Wake_Up_Sign } \\
\text { al }\end{array}$ & $\begin{array}{c}\text { ResTW, } \\
\text { LID:=SavedLID, } \\
\text { SAVE:=SavedSAVE }\end{array}$ & DATA_1 \\
\cline { 2 - 5 } & 2 & TWait_expired & $\begin{array}{c}\text { SavedState:=WAIT, } \\
\text { PowDown }\end{array}$ & SLEEP \\
\hline
\end{tabular}

All LID in UL frames are private. $\mathrm{M}$ in RSE/OBE indicates that it is mandatory to support the frame. The opt in OBE indicates that it is optional to support the frame.

TABLE II. UL FRAMES SPECIFICATION

\begin{tabular}{|c|c|c|c|c|c|c|}
\hline $\begin{array}{l}\mathrm{n} \\
\mathbf{0}\end{array}$ & $\begin{array}{c}\text { MA } \\
\text { C }\end{array}$ & $\begin{array}{c}\mathbf{L L} \\
\mathrm{C}\end{array}$ & $\begin{array}{c}\text { LL } \\
\text { C } \\
\text { stat } \\
\text { us }\end{array}$ & APDU & $\begin{array}{c}\text { window } \\
\text { request }(\mathrm{WR})\end{array}$ & $\begin{array}{l}\text { RSE/ } \\
\text { OBE }\end{array}$ \\
\hline 1 & 60 & $\begin{array}{l}\text { no } \\
\text { ne }\end{array}$ & $\begin{array}{c}\text { non } \\
\mathrm{e}\end{array}$ & none & $\begin{array}{l}\text { Private UL WR } \\
\text { (in public UL } \\
\text { window only) }\end{array}$ & $\mathrm{M} / \mathrm{M}$ \\
\hline 2 & $\mathrm{CO}$ & 03 & $\begin{array}{c}\text { non } \\
\mathrm{e}\end{array}$ & $\begin{array}{l}\text { INIT.response } \\
\text { (VST) }\end{array}$ & $\begin{array}{c}\text { Private UI } \\
\text { command, No } \\
\text { WR }\end{array}$ & $\mathrm{M} / \mathrm{M}$ \\
\hline 3 & $\mathrm{C} 0$ & 03 & $\begin{array}{c}\text { non } \\
\mathrm{e}\end{array}$ & $\begin{array}{c}\text { GET. response, } \\
\text { SET. response, } \\
\text { ACTION. } \\
\text { response }\end{array}$ & $\begin{array}{c}\text { Private UI } \\
\text { command, No } \\
\text { WR }\end{array}$ & M/opt \\
\hline 4 & D0 & $\begin{array}{l}67 / \\
\text { E7 }\end{array}$ & 40 & $\begin{array}{l}\text { None, } \\
\text { (APDU not } \\
\text { requested) }\end{array}$ & $\begin{array}{c}\text { Private } \mathrm{ACn} \\
\text { response } \mathrm{f}=0 \text {, } \\
\text { No } \mathrm{WR}\end{array}$ & $\mathrm{M} / \mathrm{M}$ \\
\hline 5 & D0 & $\begin{array}{l}77 / \\
\text { F7 }\end{array}$ & 30 & $\begin{array}{c}\text { None, } \\
\text { (APDU not } \\
\text { available) }\end{array}$ & $\begin{array}{c}\text { Private } \mathrm{ACn} \\
\text { response } \mathrm{f}= \\
1, \text { No WR }\end{array}$ & M/opt \\
\hline 6 & D0 & $\begin{array}{l}77 / \\
\text { F7 }\end{array}$ & 00 & $\begin{array}{l}\text { GET. response, } \\
\text { SET. response, }\end{array}$ & $\begin{array}{c}\text { Private } \mathrm{ACn} \\
\text { response } \mathrm{f}=1,\end{array}$ & $\mathrm{M} / \mathrm{M}$ \\
\hline
\end{tabular}

\begin{tabular}{|l|l|l|l|l|l|l|}
\hline & & & $\begin{array}{c}\text { ACTION. } \\
\text { response }\end{array}$ & No WR & \\
\hline
\end{tabular}

\section{SUMMARIES}

Korea ETRI has participated the ITU-T SG16 (Vehicle Gateway Platform) standards project since 2009. Taiwan Bureau of Standards, Metrology \& Inspection (BSMI) also works on the DSRC standards. DSRC provides the platform for interoperable ETC and access control of various applications. DSRC specification is responsive to both customer needs and market requirements. Key features of this research include an understanding of stability and interoperability that offer value to customers.

\section{REFERENCES}

[1] CEN/TC278, prEN 12253 "Road Transport and Traffic Telematics Dedicated Short Range Communication (DSRC) - DSRC Physical Layer using Microwave at $5.8 \mathrm{GHz}$ " - Jan 2003

[2] CEN/TC278, EN 12795 "Road Transport and Traffic Telematics Dedicated Short Range Communication (DSRC) - DSRC Data Link Layer: Medium Access and Logical Link Control"

[3] CEN/TC278, EN 12834 "Road Transport and Traffic Telematics Dedicated Short Range Communication (DSRC) - DSRC Application Layer"

[4] Global Specification for Short Range Communication, The platform for Interoperable ETC and Access Control, http://profesores.elo.utfsm.cl/ agv/elo326/1s06/ETC/GSS_32.pdf, available on 20110504

[5] ISO 14096 -2004 Road transport and traffic telematics -Electronic fee collection - Application interface definition for dedicated shortrange communication Design of GPS-Based System to Avoid CamelVehicle Collisions: A Review, Mohammed S. Zahrani, Khaled Ragab and Asrar Ul Haque, http://scialert.net/abstract/?doi=ajaps.2011.362.377, available on 20110504

[6] CEN/TC278, prEN 13372 "Road Transport and Traffic Telematics Dedicated Short Range Communication (DSRC) - DSRC Profiles for RTTT Applications" - Jan 2003

[7] Tien-chen, Ho, The Study of Intelligent telematics technology evolution, Information Technology Dept., OCU, accepted on 2011/06/03, http://ilt2011.ncut.edu.tw/

[8] www.its-taiwan.org.tw/download/downlo, available on 20110504

[9] www.iot.gov.tw/public/Attachment/4722, available on 20110504 